九州大学学術情報リポジトリ

Kyushu University Institutional Repository

\title{
REMARKS ON IDEAL COMPLETION OF *-CONTINUOUS IDEMPOTENT LEFT SEMIRINGS
}

\section{Furusawa, Hitoshi}

Department of Mathematics and Computer Science, Graduate School of Science and Engineering, Kagoshima University

Sanda, Fumiya

Chosa Junior High School

Tsumagari, Norihiro

Department of System Information Science, Graduate School of Science and Engineering, Kagoshima University | Japan Society for the Promotion of Science : Research Fellow

https://doi.org/10.5109/1434308

出版情報: Bulletin of informatics and cybernetics. 43, pp.1-21，2011-12. Research Association of Statistical Sciences

バージョン :

権利関係 : 
REMARKS ON IDEAL COMPLETION OF *-CONTINUOUS IDEMPOTENT LEFT SEMIRINGS

by

Hitoshi Furusawa, Fumiya Sanda and Norihiro Tsumagari

Reprinted from the Bulletin of Informatics and Cybernetics Research Association of Statistical Sciences, Vol.43

FUKUOKA, JAPAN

2011 


\title{
REMARKS ON IDEAL COMPLETION OF *-CONTINUOUS IDEMPOTENT LEFT SEMIRINGS
}

\author{
By
}

Hitoshi Furusawa* Fumiya Sanda $^{\dagger}$ and Norihiro TsumagarI ${ }^{\ddagger}$

\begin{abstract}
It has been reported that the set of $*$-ideals of a $*$-continuous idempotent left semiring forms a D-continuous idempotent left semiring and the construction satisfies a universal property. However, we have found a $*$-continuous idempotent left semiring that the set of its $*$-ideals never forms a D-continuous idempotent left semiring. In this paper, we give this example and show that the reported properties hold if a $*$-continuous idempotent left semiring satisfies left distributivity.
\end{abstract}

Key Words and Phrases: *-continuous Idempotent Left Semirings, D-continuous Idempotent Left Semirings, $*$-ideals, Ideal Completion

\section{Introduction}

A notion of Kleene algebras is introduced by Kozen (1994) as a complete axiomatisation of regular events. Considering that regular expressions appears everywhere in fields of computer science, it may not be hard to presume that a Kleene algebra can capture properties of structures appeared in the fields.

A Kleene algebra is an idempotent semirings with a unary operation *. An idempotent semiring is a tuple $(S,+, \cdot, 0,1)$ consisting of a set $S$, two binary operations + and on $S$, and two elements 0 and 1 of $S$ such that $(S,+, 0)$ is a commutative and idempotent monoid, $(S, \cdot, 1)$ is a monoid, and $\cdot$ distributes over + from the both sides and 0 is a left and right zero element of $\cdot$. So, $(S,+)$ is a semilattice whose ordering is defined by $a \leq b$ iff $a+b=b$ for all $a, b \in S$. Thus the sum $a+b$ of two elements $a$ and $b$ is the join or the least upper bound of $\{a, b\}$ and 0 is the least element with respect to $\leq$. An idempotent semiring with a unary operation * is called Kleene algebra if the unary operation * satisfies the following four conditions.

$$
\begin{aligned}
1+a a^{*} \leq a^{*} \\
1+a^{*} a \leq a^{*} \\
a x \leq x \rightarrow a^{*} x \leq x \\
x a \leq x \rightarrow x a^{*} \leq x
\end{aligned}
$$

\footnotetext{
* Department of Mathematics and Computer Science, Graduate School of Science and Engineering, Kagoshima University. furusawa@sci.kagoshima-u.ac.jp

† Chosa Junior High School. k6618413@kadai.jp

¥ Department of System Information Science, Graduate School of Science and Engineering, Kagoshima University. Research Fellow of the Japan Society for the Promotion of Science. k1852610@kadai.jp
} 
A Kleene algebra is called $*$-continuous if it satisfies the axiom

$$
a b^{*} c=\sum_{n \geq 0} a b^{n} c
$$

where $\sum$ refers to the least upper bounds in the natural order $\leq$. In fact, (5) implies (1)-(4) in each idempotent semiring. Thus, $*$-continuous Kleene algebras might be called *-continuous idempotent semirings. S-algebras introduced by Conway (1971) are idempotent semirings with arbitrary summation operation $\sum$ and their binary operation . distributes over $\sum$ from the both sides. Thus, S-algebras might be called complete idempotent semirings. Conway (1971) has given a construction that embeds every *continuous Kleene algebra into an S-algebra. This construction can be described as an ideal completion. Kozen (1990) has shown that the construction satisfies a universal property.

Recently, weaker variants of Kleene algebras have been introduced to capture properties which may not be handled in Kleene algebras. Studying difference and similarity of Kleene algebras and their weaker variants is necessary to make use of these.

Analogous results to ones shown by Kozen (1990) on variants of lazy Kleene algebras (cf. Möller (2004)) that are idempotent left semirings with a unary operation * satisfying *-continuity has been reported by Furusawa and Sanda (2009). In order to investigate weaker structures than the case of Kozen (1990), improved notions of *continuity and $*$-ideals have been introduced. Though (5) implies (1)-(4) even in each idempotent left semiring, *-continuous idempotent left semirings satisfy (1) and (3). The notion of $*$-ideals of $*$-continuous idempotent left semirings is compatible with the notion of $*$-continuity for idempotent left semirings rather than (5). However, some of results by Furusawa and Sanda (2009) are wrong. In this paper, we point out its errors with examples. To give them, we use restricted tree languages called monodic tree languages. Also, we sort out correct results from wrong ones.

\section{Idempotent Left Semirings}

Idempotent left semirings (cf. Möller (2004)) are defined as follows.

Definition 2.1. An idempotent left semiring, or briefly an IL-semiring is a tuple $(S,+, \cdot, 0,1)$ with a set $S$, two binary operations + and $\cdot$, and $0,1 \in S$ satisfying the following properties:

- $(S,+, 0)$ is an idempotent commutative monoid.

- $(S, \cdot, 1)$ is a monoid.

- For all $a, b, c \in S, a \cdot c+b \cdot c=(a+b) \cdot c, a \cdot b+a \cdot c \leq a \cdot(b+c)$, and $0 \cdot a=0$,

where the natural order $\leq$ is given by $a \leq b$ iff $a+b=b$.

The natural order $\leq$ on an IL-semiring is a join semilattice with the least element 0 , where its join operation is given by the binary summation operation + . In this paper, a directed subset of an idempotent left semiring stands for a nonempty subset whose each finite subset has an upper bound in itself. We often abbreviate $a \cdot b$ to $a b$. 
REMARK 2.2. An IL-semiring $S$ satisfying $a b+a c=a(b+c)$ and $a 0=0$ for all $a, b, c \in S$ is an idempotent semiring.

Let $S$ be an IL-semiring. For $a \in S$ and a natural number $n, a^{n}$ and $a^{(n)}$ are respectively defined by the following induction.

- $a^{0}=1, a^{n+1}=a a^{n}$.

- $a^{(0)}=0, a^{(n+1)}=a a^{(n)}+1$.

Note that $a^{(n)} \leq a^{(n+1)}$.

LEMma 2.3. Let $S$ be an IL-semiring and $a, b \in S$. Then, for each natural number $n \geq 0$,

1. $a b \leq b$ implies $a^{(n)} b \leq b$, and

2. $b(a+1) \leq b$ implies $b a^{(n)} \leq b$.

Proof. These two are proved by induction on $n \geq 0$. For $n=0, a^{(0)} b=0 \leq b$. Assume that $a^{(n)} b \leq b$. Then, the following holds.

$$
\begin{array}{rlrl}
a^{(n+1)} b & =\left(a a^{(n)}+1\right) b & \\
& =a a^{(n)} b+b & & \\
& \leq a b+b & & \text { (by induction hypothesis) } \\
& \leq b & & \text { (by } a b \leq b)
\end{array}
$$

For $n=0, b a^{(0)}=b 0 \leq b$. For $n=1, b a^{(1)}=b(a 0+1) \leq b(a+1) \leq b$. Assume that $b a^{(n+1)} \leq b$ and note that $1 \leq a a^{(n)}+1=a^{(n+1)}$. Then, the following holds.

$$
\begin{aligned}
b a^{(n+2)} & =b\left(a a^{(n+1)}+1\right) & & \\
& \leq b\left(a a^{(n+1)}+a^{(n+1)}\right) & & (\text { by notice) } \\
& =b(a+1) a^{(n+1)} & & \\
& \leq b a^{(n+1)} & & \text { (by } b(a+1) \leq b) \\
& \leq b & & \text { (by induction hypothesis) }
\end{aligned}
$$

For $A \subseteq S, \sum A$ denotes the join or the least upper bound of $A$ with respect to the natural order $\leq$ on $S$ if it exists.

One of the true purposes of introducing $\sum$ and ${ }^{(n)}$ is to axiomatise a unary operation $*$ in the following definition.

Definition 2.4. A *-continuous IL-semiring is a tuple $\left(S,+, \cdot,{ }^{*}, 0,1\right)$ with a set $S$, two binary operations + and $\cdot$, a unary operation *, and $0,1 \in S$ satisfying the following properties:

- $(S,+, \cdot, 0,1)$ is an IL-semiring.

- For all $a, b, c \in S$, the least upper bound of $\left\{a b^{(n)} c \mid n \geq 0\right\}$ exists in $S$.

- $a b^{*} c=\sum_{n \geq 0} a b^{(n)} c$ holds for all $a, b, c \in S$. 
A homomorphism between $*$-continuous IL-semirings is a mapping between their underlying sets, which preserves $+, \cdot{ }^{*}, 0$ and 1 .

Replacing $a$ and $c$ with 1 , we have $b^{*}=\sum_{n \geq 0} b^{(n)}$.

REMARK 2.5. The natural ordering on a $*$-continuous IL-semiring need not be complete. It is known that the set of regular languages on an alphabet is a $*$-continuous IL-semiring (Kleene algebra) together with union, concatenation, Kleene-star, empty language, and a singleton set of empty word. Consider the set of regular language on $\{a, b\}$. Then, $\bigcup\left\{\left\{a^{n} b^{n}\right\} \mid n \geq 0\right\}$ is not a regular language on $\{a, b\}$ even though $\left\{a^{n} b^{n}\right\}$ is a regular language on the alphabet for each natural number $n$.

We write ILS* for the category whose objects are *-continuous IL-semirings and whose arrows are homomorphisms between them. ILS* $\left(S, S^{\prime}\right)$ denotes the set of arrows of ILS* from $S$ to $S^{\prime}$. See, for example, Barr and Wells (1999) or Mac Lane (1998) for details on category theory.

A lazy Kleene algebra is an IL-semiring with a unary operation * satisfying the following two conditions.

$$
\begin{aligned}
1+a a^{*} & \leq a^{*} \\
a b \leq b & \rightarrow a^{*} b \leq b
\end{aligned}
$$

The following property shows that a $*$-continuous IL-semiring is a lazy Kleene algebra satisfying the D-axiom (cf. Nishizawa et al. (2009)) (or a monodic tree Kleene algebra (cf. Takai and Furusawa (2006))).

Proposition 2.6. A *-continuous $I L$-semiring $S$ satisfies the following.

1. $1+a a^{*} \leq a^{*}$.

2. $a b \leq b$ implies $a^{*} b \leq b$.

3. $b(a+1) \leq b$ implies $b a^{*} \leq b$ (the $D$-axiom).

Proof. Note that $1 \leq a^{*}$ since

$$
1 \leq a 0+1 \leq \sum_{n \geq 0} a^{(n)}=a^{*} .
$$

Also $a a^{*} \leq a^{*}$ since

$$
a a^{*}=\sum_{n \geq 0} a a^{(n)} \text { and } a a^{(k)} \leq a a^{(k)}+1=a^{(k+1)} \leq \sum_{n \geq 0} a^{(n)}=a^{*}
$$

for each $k \geq 0$. So we have $1+a a^{*} \leq a^{*}$. Suppose $a b \leq b$. Then we have

$$
a^{*} b=\sum_{n \geq 0} a^{(n)} b \leq b
$$

by Lemma 2.3. Similarly, it holds that $b(a+1) \leq b$ implies $b a^{*} \leq b$. 
Definition 2.7. A *-continuous IL-semiring satisfying the right zero law (or the 0-axiom (cf. Nishizawa et al. (2009))) is a $*$-continuous IL-semiring satisfying

$$
a 0=0
$$

for every element $a$. A $*$-continuous IL-semiring satisfying left distributivity (or the +-axiom (cf. Nishizawa et al. (2009))) is a $*$-continuous IL-semiring satisfying

$$
a b+a c=a(b+c)
$$

for all elements $a, b$, and $c$.

We write

- ILS* for the full subcategory of ILS*, whose objects are $*$-continuous IL-semirings satisfying the right zero law, and $\operatorname{ILS}_{0}^{*}\left(S, S^{\prime}\right)$ for the set of arrows of ILS $S_{0}^{*}$ from $S$ to $S^{\prime}$,

- ILS* for the full subcategory of ILS*, whose objects are $*$-continuous IL-semirings satisfying left distributivity, and ILS* $\left(S, S^{\prime}\right)$ for the set of arrows of ILS* from $S$ to $S^{\prime}$, and

- ILS ${ }_{0,+}^{*}$ for the full subcategory of ILS*, whose objects are $*$-continuous IL-semirings satisfying both of the right zero law and left distributivity, and $\operatorname{ILS}_{0,+}^{*}\left(S, S^{\prime}\right)$ for the set of arrows of ILS ${ }_{0,+}^{*}$ from $S$ to $S^{\prime}$,.

The following is immediate from Proposition 2.6.

Corollary 2.8. Let $S$ be a $*$-continuous IL-semiring.

- If $S$ satisfies the right zero law, $S$ is a lazy Kleene algebra satisfying the D-axiom and the 0-axiom (or a probabilistic Kleene algebra (cf. McIver and Weber (2005))).

- If $S$ satisfies left distributivity, $S$ is a lazy Kleene algebra satisfying the D-axiom and the +-axiom.

- If $S$ satisfies the right zero law and left distributivity, $S$ is a lazy Kleene algebra satisfying the D-axiom, the 0-axiom, and the +-axiom (or a Kleene algebra (cf. Kozen (1990), Kozen (1994))).

REMARK 2.9. If $S$ is a $*$-continuous IL-semiring satisfying left distributivity, then

$$
a^{(n+1)}=a^{n+1} 0+\sum_{0 \leq k \leq n} a^{k}
$$

holds for each $a \in S$ and natural number $n$. Thus,

$$
\sum_{0 \leq k \leq n} a^{k} \leq a^{(n+1)} \leq \sum_{0 \leq k \leq n+1} a^{k}
$$

Therefore,

$$
a^{*}=\sum_{n \geq 0} a^{(n)}=\sum_{n \geq 0} a^{n}
$$

So, a $*$-continuous IL-semiring $S$ satisfying the right zero law and left distributivity is a *-continuous Kleene algebra (cf. Kozen (1990)). 
Kozen (1990) has given an example of a Kleene algebra which is not $*$-continuous. The example shows that the converse statements of Proposition 2.6 and Corollary 2.8 need not hold.

Definition 2.10. A complete IL-semiring $S$ is an IL-semiring satisfying the following properties: For each $A \subseteq S$,

- the least upper bound $\sum A$ of $A$ exists in $S$, and

- $\left(\sum A\right) a=\sum\{x a \mid x \in A\}$ for each $a \in S$.

A D-continuous IL-semiring (or a complete IL-semiring preserving right directed joins (cf. Nishizawa et al. (2009))) $S$ is a complete IL-semiring satisfying

$$
a \sum A=\sum\{a x \mid x \in A\}
$$

for each $a \in S$ and each directed subset $A \subseteq S$. A homomorphism between D-continuous IL-semirings is a mapping between underlying sets, which preserves $+, \cdot, 0,1$, and arbitrary $\sum$. A D-continuous IL-semiring satisfying the right zero law (or preserving the right 0 (cf. Nishizawa et al. (2009))) is a D-continuous IL-semiring satisfying

$$
a 0=0
$$

for each element $a$. A D-continuous IL-semiring satisfying left distributivity (or preserving the right $+($ cf. Nishizawa et al. (2009))) is a D-continuous IL-semiring satisfying

$$
a b+a c=a(b+c)
$$

for any elements $a, b$, and $c$.

REMARK 2.11 (Nishizawa et al. (2009)). Let $Q$ be a D-continuous IL-semiring.

1. $Q$ satisfies left distributivity iff $Q$ is a complete IL-semiring whose binary operation - distributes over all non-empty joins even from the left hand side.

2. $Q$ satisfies the right zero law and left distributivity iff $Q$ is an $\mathbf{S}$-algebra or a complete idempotent semiring.

We write

- ILS ${ }^{D}$ for the category whose objects are D-continuous IL-semirings and whose arrows are homomorphisms between them, and $\operatorname{ILS}^{D}\left(S, S^{\prime}\right)$ for the set of arrows of ILS ${ }^{D}$ from $S$ to $S^{\prime}$,

- ILS ${ }_{0}^{D}$ for the full subcategory of ILS ${ }^{D}$, whose objects are D-continuous IL-semirings satisfying the right zero law, and $\operatorname{ILS}_{0}^{D}\left(S, S^{\prime}\right)$ for the set of arrows of ILS ${ }_{0}^{D}$ from $S$ to $S^{\prime}$,

- ILS ${ }_{+}^{D}$ for the full subcategory of ILS ${ }^{D}$, whose objects are D-continuous IL-semirings satisfying left distributivity, and $\operatorname{ILS}_{+}^{D}\left(S, S^{\prime}\right)$ for the set of arrows of ILS $S_{+}^{D}$ from $S$ to $S^{\prime}$, and 
- ILS $_{0,+}^{D}$ for the full subcategory of ILS ${ }^{D}$, whose objects are D-continuous IL-semirings satisfying both of the right zero law and left distributivity, and $\operatorname{ILS}_{0,+}^{D}\left(S, S^{\prime}\right)$ for the set of arrows of ILS $0_{0,+}^{D}$ from $S$ to $S^{\prime}$.

Let $Q$ and $Q^{\prime}$ be objects and $f: Q \rightarrow Q^{\prime}$ an arrow of $\operatorname{ILS}^{D}$. For each $a \in Q$, defining

$$
a^{*}=\sum_{n \geq 0} a^{(n)}
$$

it holds that

$$
b a^{*} c=\sum_{n \geq 0} b a^{(n)} c
$$

since the set $\left\{a^{(n)} \mid n \geq 0\right\}$ is a directed subset of $Q$. Also, $f$ preserves ${ }^{*}$ since $f$ preserves $+, \cdot \sum, 0,1$, and ${ }^{*}$ is defined using these operations. Therefore, the following holds.

Proposition 2.12. Each D-continuous IL-semiring is a *-continuous $I L$-semiring. Moreover, ILS ${ }^{D}$ is a subcategory of ILS*.

We write $G:$ ILS $^{D} \rightarrow$ ILS $^{*}$ for the inclusion functor from ILS ${ }^{D}$ to ILS*. The following is immediate from Proposition 2.12.

Corollary 2.13. Let $Q$ be a D-continuous IL-semiring.

- If $Q$ satisfies the right zero law, $Q$ is a *-continuous IL-semiring satisfying the right zero law. Moreover, $\mathrm{ILS}_{0}^{D}$ is a subcategory of $\mathrm{ILS}_{0}^{*}$.

- If $Q$ satisfies left distributivity, $Q$ is a *-continuous IL-semiring satisfying left distributivity. Moreover, ILS ${ }_{+}^{D}$ is a subcategory of ILS ${ }_{+}^{*}$.

- If $Q$ satisfies the right zero law and left distributivity, $Q$ is a *-continuous $I L$ semiring satisfying the right zero law and left distributivity. Moreover, $\operatorname{ILS}_{0,+}^{D}$ is a subcategory of $\mathrm{ILS}_{0,+}^{*}$.

We write

- $G_{0}: \mathrm{ILS}_{0}^{D} \rightarrow \mathrm{ILS}_{0}^{*}$ for the inclusion functor from $\mathrm{ILS}_{0}^{D}$ to $\mathrm{ILS}_{0}^{*}$,

- $G_{+}: \mathrm{ILS}_{+}^{D} \rightarrow \mathrm{ILS}_{+}^{*}$ for the inclusion functor from ILS $\mathrm{IL}_{+}^{D}$ to ILS $\mathrm{IL}_{+}^{*}$, and

- $G_{0,+}: \mathrm{ILS}_{0,+}^{D} \rightarrow \mathrm{ILS}_{0,+}^{*}$ for the inclusion functor from $\mathrm{ILS}_{0,+}^{D}$ to ILS ${ }_{0,+}^{*}$.

\section{D-continuous IL-semirings and Monodic Tree Languages}

A ranked set is a couple $(\Sigma, r)$ of a set $\Sigma$ of (function) symbols and a mapping $r$ from $\Sigma$ to the set $\mathbf{N}$ of natural numbers. We often abbreviate $(\Sigma, r)$ to $\Sigma$. $\Sigma_{n}$ denotes the set $\{a \in \Sigma \mid r(a)=n\}$ of symbols of rank $n$. The set $T_{\Sigma}$ of terms over $\Sigma$ is defined by the following induction:

- $\square \in T_{\Sigma}(\square \notin \Sigma)$,

- $\Sigma_{0} \subseteq T_{\Sigma}$ 
- for $a \in \Sigma_{n}$ and $t_{1}, \cdots, t_{n} \in T_{\Sigma}, a\left(t_{1}, \cdots, t_{n}\right) \in T_{\Sigma}$.

A subset of $T_{\Sigma}$ is called monodic tree language over $\Sigma$. Obviously, $\left(\wp\left(T_{\Sigma}\right), \cup, \emptyset\right)$ is an idempotent commutative monoid.

REMARK 3.1. In the case of $\Sigma=\Sigma_{1}, T_{\Sigma}$ is isomorphic to the set $\Sigma^{*}$ of finite strings over $\Sigma$

For $t \in T_{\Sigma}$ and $L \subseteq T_{\Sigma}$, we define $t \cdot L \subseteq T_{\Sigma}$ as follows:

- if $t=\square$, then $t \cdot L=L$,

- if $t \in \Sigma_{0}$, then $t \cdot L=\{t\}$,

- if $t=a\left(t_{1}, \cdots, t_{n}\right)$, then $t \cdot L=\left\{a\left(u_{1}, \cdots, u_{n}\right) \mid u_{i} \in t_{i} \cdot L\right\}$.

Lemma 3.2. Let $t \in T_{\Sigma}, L, L^{\prime} \subseteq T_{\Sigma}$, and $\mathcal{L} \subseteq \wp\left(T_{\Sigma}\right)$.

1. $t \cdot\{\square\}=\{t\}$.

2. $t \cdot L \subseteq t \cdot L^{\prime}$ if $L \subseteq L^{\prime}$.

3. $t \cdot(\bigcup \mathcal{L})=\bigcup\left\{t \cdot L^{\prime} \mid L^{\prime} \in \mathcal{L}\right\}$ if $\mathcal{L}$ is a directed subset with respect to the inclusion ordering $\subseteq$.

Proof. 1 and 2 are shown by induction on the construction of $t$. By 2 ,

$$
\bigcup\left\{t \cdot L^{\prime} \mid L^{\prime} \in \mathcal{L}\right\} \subseteq t \cdot(\bigcup \mathcal{L}) .
$$

The reverse inclusion is shown by induction on the construction of $t$. In the case of $t=\square$ and $t \in \Sigma_{0}$, it holds obviously. We consider the case of $t=a\left(t_{1}, \cdots, t_{n}\right)$. Assume that $t_{i} \cdot(\bigcup \mathcal{L}) \subseteq \bigcup\left\{t_{i} \cdot L^{\prime} \mid L^{\prime} \in \mathcal{L}\right\}$ for each $i \in\{1, \cdots, n\}$. Then, we have

$$
\begin{array}{ll}
a\left(u_{1}, \cdots, u_{n}\right) \in t \cdot(\bigcup \mathcal{L}) & \\
\Longleftrightarrow \forall i \in\{1, \cdots, n\} \cdot u_{i} \in t_{i} \cdot(\bigcup \mathcal{L}) & \\
\Longrightarrow \forall i \in\{1, \cdots, n\} \cdot \exists L_{i} \in \mathcal{L} \cdot u_{i} \in t_{i} \cdot L_{i} & \text { (by induction hypothesis) } \\
\Longrightarrow \exists L \in \mathcal{L} \cdot \forall i \in\{1, \cdots, n\} \cdot u_{i} \in t_{i} \cdot L & \text { (since } \mathcal{L} \text { is directed) } \\
\Longleftrightarrow \exists L \in \mathcal{L} \cdot a\left(u_{1}, \cdots, u_{n}\right) \in t \cdot L & \\
\Longleftrightarrow a\left(u_{1}, \cdots, u_{n}\right) \in \bigcup\left\{t \cdot L^{\prime} \mid L^{\prime} \in \mathcal{L}\right\} . &
\end{array}
$$

Defining, for $L, L^{\prime} \subseteq T_{\Sigma}$,

$$
L \cdot L^{\prime}=\bigcup\left\{t \cdot L^{\prime} \mid t \in L\right\},
$$

$L \cdot L^{\prime} \subseteq T_{\Sigma}$ and $\cdot$ is extended to a binary operation on $\wp\left(T_{\Sigma}\right)$. By the definition, it holds that $\{\square\} \cdot L=L$ and $\emptyset \cdot L=\emptyset$. Also, by 1 and 2 of Lemma 3.2, it holds that $L \cdot\{\square\}=L$ and $L \cdot L^{\prime} \subseteq L \cdot L^{\prime \prime}$ if $L^{\prime} \subseteq L^{\prime \prime}$.

Lemma 3.3. Let $L, L^{\prime}, L^{\prime \prime} \subseteq T_{\Sigma}$ and $\mathcal{L} \subseteq \wp\left(T_{\Sigma}\right)$. 
1. $(\bigcup \mathcal{L}) \cdot L=\bigcup\left\{L^{\prime} \cdot L \mid L^{\prime} \in \mathcal{L}\right\}$.

2. $\left(L \cdot L^{\prime}\right) \cdot L^{\prime \prime}=L \cdot\left(L^{\prime} \cdot L^{\prime \prime}\right)$.

Proof. 1 follows from

$$
\begin{aligned}
t \in(\bigcup \mathcal{L}) \cdot L & \Longleftrightarrow \quad \exists t^{\prime} \in \bigcup \mathcal{L} \cdot t \in t^{\prime} \cdot L \\
& \Longleftrightarrow \quad \exists L^{\prime} \in \mathcal{L} \cdot \exists t^{\prime} \in L^{\prime} \cdot t \in t^{\prime} \cdot L \\
& \Longleftrightarrow \quad \exists L^{\prime} \in \mathcal{L} \cdot t \in L^{\prime} \cdot L \\
& \Longleftrightarrow t \in \bigcup\left\{L^{\prime} \cdot L \mid L^{\prime} \in \mathcal{L}\right\}
\end{aligned}
$$

By induction on the construction of $t$, it is shown that $\left(t \cdot L^{\prime}\right) \cdot L^{\prime \prime}=t \cdot\left(L^{\prime} \cdot L^{\prime \prime}\right)$ for each $t \in T_{\Sigma}$ and $L^{\prime}, L^{\prime \prime} \subseteq T_{\Sigma}$. Noting this fact, 2 follows from

$$
\begin{aligned}
\left(L \cdot L^{\prime}\right) \cdot L^{\prime \prime} & =\left(\left\{t \cdot L^{\prime} \mid t \in L\right\}\right) \cdot L^{\prime \prime} \\
& \left.=\bigcup\left\{\left(t \cdot L^{\prime}\right) \cdot L^{\prime \prime} \mid t \in L\right\} \quad \text { (by } 1\right) \\
& =\bigcup\left\{t \cdot\left(L^{\prime} \cdot L^{\prime \prime}\right) \mid t \in L\right\} \quad \text { (by notice) } \\
& =L \cdot\left(L^{\prime} \cdot L^{\prime \prime}\right) .
\end{aligned}
$$

From the discussion above, the following property holds. and .

Proposition 3.4. $\wp\left(T_{\Sigma}\right)$ forms a complete IL-semiring together with $\cup, \cdot, \emptyset,\{\square\}$,

REMARK 3.5. It is known that $\wp\left(A^{*}\right)$ is a complete idempotent semiring freely generated by a set $A$. Therefore, in the case of $\Sigma=\Sigma_{1}, \wp\left(T_{\Sigma}\right)$ is a complete idempotent semiring freely generated by $\Sigma$.

Also, $\wp\left(T_{\Sigma}\right)$ forms a D-continuous IL-semiring, but the right zero law and left distributivity need not be satisfied.

Theorem 3.6. $\wp\left(T_{\Sigma}\right)$ forms a D-continuous IL-semiring. Moreover, for the Dcontinuous IL-semiring $\wp\left(T_{\Sigma}\right)$,

1. the right zero law is satisfied iff $\Sigma_{0}=\emptyset$, and

2. left distributivity is satisfied iff $\Sigma_{n}=\emptyset$ for each $n \geq 2$.

Proof. By Proposition 3.4 and 3 of Lemma 3.2, $\wp\left(T_{\Sigma}\right)$ forms a $D$-continuous IL-semiring. Suppose that $\Sigma_{0}=\emptyset$. Then, by induction on the construction of $t \in T_{\Sigma}$, $t \cdot \emptyset=\emptyset$. Thus, the right zero law holds. For the inverse, we suppose that $\Sigma_{0} \neq \emptyset$. Then, we have $c \in \Sigma_{0}$ and $\{c\} \cdot \emptyset=\{c\}$. Therefore, 1 holds. Next, suppose that $\Sigma_{n}=\emptyset$ for each $n \geq 2$. Then, it holds that $t \cdot\left(L \cup L^{\prime}\right)=t \cdot L \cup t \cdot L^{\prime}$ since $t \in T_{\Sigma}$ is either $\square$, an element of $\Sigma_{0}$, or a string over $\Sigma_{1}$ whose leaf is $\square$. Thus left distributivity holds. For the inverse, we suppose that there exists $n$ such that $n \geq 2$ and $\Sigma_{n} \neq \emptyset$. Take $a \in \Sigma_{n}$. Then, $a(\square, \cdots, \square) \in T_{\Sigma}$. For $t, t^{\prime} \in T_{\Sigma}$ such that $t \neq t^{\prime}$, we have

$$
a(\square, \cdots, \square) \cdot\{t\} \cup a(\square, \cdots, \square) \cdot\left\{t^{\prime}\right\} \subsetneq a(\square, \cdots, \square) \cdot\left(\{t\} \cup\left\{t^{\prime}\right\}\right) .
$$

Therefore, 2 holds. 
It is immediate from the above theorem that $\wp\left(T_{\Sigma}\right)$ forms a $*$-continuous IL-semiring. Also, for the $*$-continuous IL-semiring $\wp\left(T_{\Sigma}\right)$,

1. the right zero law is satisfied iff $\Sigma_{0}=\emptyset$, and

2. left distributivity is satisfied iff $\Sigma_{n}=\emptyset$ for each $n \geq 2$.

\section{4. $*$-ideals}

Conway (1971) introduced $*$-ideals for $*$-continuous Kleene algebras. *-ideals for *-continuous IL-semirings are defined by Furusawa and Sanda (2009) as follows.

Definition 4.1. Let $S$ be a $*$-continuous IL-semiring. A $*$-ideal is a subset $A$ of $S$ such that

- $A$ is nonempty,

- $A$ is closed under + ,

- $A$ is closed downward under $\leq$,

- if $a b^{(n)} c \in A$ for all $n \geq 0$, then $a b^{*} c \in A$.

Let $S$ be a $*$-continuous IL-semiring. For a subset $A \subseteq S$, define

$$
A \downarrow=\{y \mid \exists x \in A . y \leq x\} .
$$

ExAmple 4.2. Set $\Sigma_{1}=\{b, c\}, \Sigma_{2}=\{a\}$, and $\Sigma=\Sigma_{1} \cup \Sigma_{2}$. Then, $\wp\left(T_{\Sigma}\right)$ forms a *-continuous IL-semiring (satisfying the right zero law). Let

$$
\begin{aligned}
& M_{n}=\{a(\square, \square)\} \cdot\left(\{b(\square)\}^{(n)} \cup\{c(\square)\}\right) \\
& M_{n}^{\prime}=M_{n} \cup\left\{a\left(b^{k}(\square), b^{l}(\square)\right) \mid k, l \geq 0\right\}
\end{aligned}
$$

where $b^{0}(\square)=\square$ and $b^{n+1}(\square)=b\left(b^{n}(\square)\right)$. Then, clearly, $\left\{M_{n}^{\prime} \mid n \geq 0\right\} \downarrow$ is nonempty, closed under $\cup$, and closed downward under $\subseteq$. Let $L_{1} \cdot L_{2}^{(m)} \cdot L_{3} \in\left\{M_{n}^{\prime} \mid n \geq 0\right\} \downarrow$ for all $m \geq 0$. Then, in the case of either $L_{1}, L_{2}$, or $L_{3}$ is empty or $L_{2}=\{\square\}$, it is trivial that $L_{1} \cdot L_{2}^{*} \cdot L_{3} \in\left\{M_{n}^{\prime} \mid n \geq 0\right\} \downarrow$. Otherwise, since

$$
\begin{aligned}
M_{0}^{\prime}= & \{a(c(\square), c(\square))\} \cup\left\{a\left(b^{k}(\square), b^{l}(\square)\right) \mid k, l \geq 0\right\} \\
M_{n+1}^{\prime}= & \{a(c(\square), c(\square))\} \cup\left\{a\left(b^{k}(\square), b^{l}(\square)\right) \mid k, l \geq 0\right\} \\
& \cup\left\{a\left(b^{k}(\square), c(\square)\right) \mid 0 \leq k \leq n\right\} \cup\left\{a\left(c(\square), b^{l}(\square)\right) \mid 0 \leq l \leq n\right\},
\end{aligned}
$$

for $L_{1}, L_{2}$, and $L_{3}$ such that $L_{1} \cdot L_{2}^{(m)} \cdot L_{3} \in\left\{M_{n}^{\prime} \mid n \geq 0\right\} \downarrow$,

- $t \in L_{2}$ implies neither $a$ nor $c$ occurs in $t$, i.e., $\left\{\begin{array}{l}\exists k \geq 0 . t=b^{k}(\square) \text { if } L_{2} \text { is not a singleton } \\ \exists k>0 . t=b^{k}(\square) \text { if } L_{2} \text { is a singleton }\end{array}\right.$

- $t \in L_{1}$ implies $a$ occurs only as the root of $t$ and $c$ does not occur in $t$, i.e., $\exists k, l \geq 0 . t=a\left(b^{k}(\square), b^{l}(\square)\right)$,

- $t \in L_{3}$ implies neither $a$ nor $c$ occurs in $t$, i.e., $\exists k \geq 0 . t=b^{k}(\square)$. 
Thus, if $L_{1}, L_{2}, L_{3} \neq \emptyset, L_{2} \neq\{\square\}$, and $L_{1} \cdot L_{2}^{(m)} \cdot L_{3} \in\left\{M_{n}^{\prime} \mid n \geq 0\right\} \downarrow$ for all $m \geq 0$, then $L_{1} \cdot L_{2}^{*} \cdot L_{3} \subseteq M_{0}^{\prime}$. Therefore, $L_{1} \cdot L_{2}^{*} \cdot L_{3} \in\left\{M_{n}^{\prime} \mid n \geq 0\right\} \downarrow$. From the above discussion, $\left\{M_{n}^{\prime} \mid n \geq 0\right\} \downarrow$ is a $*$-ideal.

The set of $*$-ideals of $*$-continuous IL-semiring $S$ is denoted by $\mathcal{I}(S)$. Note that $\mathcal{I}(S)$ is closed under arbitrary intersection.

We say that a nonempty set $A$ generates a $*$-ideal $I$ if $I$ is the smallest $*$-ideal containing $A .\langle A\rangle$ denotes the $*$-ideal generated by $A$. Note that $\left\langle_{-}\right\rangle$is well-defined on nonempty subsets of $S$. Also note that $\left\langle_{-}\right\rangle$is monotone and idempotent, i.e. $A \subseteq B$ implies $\langle A\rangle \subseteq\langle B\rangle$ and $\langle\langle A\rangle\rangle=\langle A\rangle$ for any nonempty subsets $A, B \subseteq S$. If $A$ is a singleton $\{a\}$, we often abbreviate $\langle\{a\}\rangle$ to $\langle a\rangle$. Such a $*$-ideal is called principal. Note that for principal ideals,

$$
\langle a\rangle=\{a\} \downarrow .
$$

Lemma 4.3. Let $S$ be a $*$-continuous $I L$-semiring. For a nonempty subset $\mathcal{A}$ of $S$,

$$
\langle\bigcup \mathcal{A}\rangle=\langle\bigcup\{\langle A\rangle \mid A \in \mathcal{A}\}\rangle \text {. }
$$

Proof. The inclusion $\subseteq$ follows from monotonicity of $\left\langle_{-}\right\rangle$. Again, by monotonicity of $\left\langle_{-}\right\rangle,\langle A\rangle \subseteq\langle\bigcup \mathcal{A}\rangle$ for each $A \in \mathcal{A}$. Thus, $\bigcup\{\langle A\rangle \mid A \in \mathcal{A}\} \subseteq\langle\bigcup \mathcal{A}\rangle$. So, we have $\langle\bigcup\{\langle A\rangle \mid A \in \mathcal{A}\}\rangle \subseteq\langle\bigcup \mathcal{A}\rangle$ by monotonicity and idempotency of $\left\langle_{-}\right\rangle$.

Let $S$ be a $*$-continuous IL-semiring. For subsets $A, B \subseteq S$, define

$$
\begin{aligned}
A \oplus B & =\{a+b \mid a \in A, b \in B\} \\
A \odot B & =\{a \cdot b \mid a \in A, b \in B\} \\
A_{*} & =\left\{a b^{*} c \mid \forall n \geq 0 . a b^{(n)} c \in A\right\} .
\end{aligned}
$$

Note that

$$
\begin{aligned}
(A \oplus B) \odot C & \subseteq(A \odot C) \oplus(B \odot C) \\
A \odot(B \downarrow) & \subseteq(A \odot B) \downarrow \\
(A \downarrow) \odot B & \subseteq(A \odot B) \downarrow \\
A \odot(B *) & \subseteq(A \odot B)_{*} \\
\left(A_{*}\right) \odot B & \subseteq(A \odot B)_{*} .
\end{aligned}
$$

REMARK 4.4 (Kozen (1990)). If $S$ satisfies left distributivity, then

$$
C \odot(A \oplus B) \subseteq(C \odot A) \oplus(C \odot B)
$$

for any subsets $A, B, C \subseteq S$.

Remark 4.11 in this paper has been shown by Kozen (1990) using transfinite induction. Following this, Lemma 4.9 was proved by transfinite induction in the previous version of this paper. According to a proof given in the referee's report, we are going to show Lemma 4.9 without using transfinite induction.

For subsets $A, B, X$ of a $*$-continuous IL-semiring $S$, define

$$
\begin{aligned}
& x \in A^{-1} X \quad \Longleftrightarrow \quad \forall a \in A . a x \in X, \\
& x \in X B^{-1} \quad \Longleftrightarrow \quad \forall b \in B . x b \in X .
\end{aligned}
$$

Note that $B \subseteq A^{-1} X$ iff $A \odot B \subseteq X$ iff $A \subseteq X B^{-1}$. Then, since $A^{-1} X \subseteq A^{-1} X$ and $X B^{-1} \subseteq X B^{-1}$, we have $A \odot A^{-1} X \subseteq X$ and $X B^{-1} \odot B \subseteq X$. Also, if $X \subseteq Y, A \subseteq A^{\prime}$, $B \subseteq B^{\prime}$, it holds that $A^{-1} X \subseteq A^{-1} Y$ and $X B^{-1} \subseteq Y B^{-1}$. 
Lemma 4.5. The following holds for subsets $X, Y, A$ and $B$ of $a$ *-continuous IL-semiring $S$.

1. $A^{-1} X \oplus A^{-1} Y \subseteq A^{-1}(X \oplus Y)$ if $S$ satisfies left distributivity.

2. $X B^{-1} \oplus Y B^{-1} \subseteq(X \oplus Y) B^{-1}$.

3. $\left(A^{-1} X\right) \downarrow \subseteq A^{-1}(X \downarrow)$.

4. $\left(X B^{-1}\right) \downarrow \subseteq(X \downarrow) B^{-1}$.

5. $\left(A^{-1} X\right)_{*} \subseteq A^{-1}\left(X_{*}\right)$.

6. $\left(X B^{-1}\right)_{*} \subseteq\left(X_{*}\right) B^{-1}$.

Proof. 1 follows from

$$
\begin{aligned}
A \odot & \left(A^{-1} X \oplus A^{-1} Y\right) \\
& \subseteq\left(A \odot A^{-1} X\right) \oplus\left(A \odot A^{-1} Y\right) \quad \text { (by Remark 4.4) } \\
& \subseteq X \oplus Y .
\end{aligned}
$$

The other inclusions are shown similarly to 1 .

The inclusion 1 of Lemma 4.5 need not hold without left distributivity.

Example 4.6. Consider the $*$-continuous IL-semiring $\wp\left(T_{\Sigma}\right)$ from Example 4.2. Set

$$
X=\{\{a(\square, \square)\} \cdot\{b(\square)\}\}, \quad Y=\{\{a(\square, \square)\} \cdot\{c(\square)\}\}, \quad A=\{\{a(\square, \square)\}\} .
$$

Then, $A^{-1} X \oplus A^{-1} Y=\{\{b(\square), c(\square)\}\}$. However, $\{b(\square), c(\square)\} \notin A^{-1}(X \oplus Y)$ since $\{a(\square, \square)\} \cdot\{b(\square), c(\square)\} \notin X \oplus Y$.

Lemma 4.7. Let $S$ be a $*$-continuous IL-semiring. The following holds for a $*$-ideal $I$ and subsets $A, B \subseteq S$.

1. $A^{-1} I$ is a $*$-ideal if $S$ satisfies left distributivity and the right zero law.

2. $I B^{-1}$ is $a *$-ideal.

Proof. 1. By the right zero law, $A \odot\{0\}=\{0\} \subseteq I$. So, $\{0\} \subseteq A^{-1} I$. Thus, $A^{-1} I$ is nonempty. Also, we have

$$
\begin{aligned}
A^{-1} I \oplus A^{-1} I & \subseteq A^{-1}(I \oplus I) \quad(\text { by } 1 \text { of Lemma } 4.5) \\
& \subseteq A^{-1} I, \\
\left(A^{-1} I\right) \downarrow & \subseteq A^{-1}(I \downarrow) \quad(\text { by } 3 \text { of Lemma } 4.5) \\
& \subseteq A^{-1} I, \\
\left(A^{-1} I\right)_{*} & \subseteq A^{-1}\left(I_{*}\right) \quad(\text { by } 5 \text { of Lemma } 4.5) \\
& \subseteq A^{-1} I .
\end{aligned}
$$

Therefore $A^{-1} I$ is a $*$-ideal. 2 is proved similarly to 1 . 
In the above lemma, the right zero law is used to prove the nonemptiness of $A^{-1} I$. So, if $A^{-1} I$ is nonempty, it is a $*$-ideal for each pair of a $*$-ideal $I$ and a subset $A$ of $S$ satisfying left distributivity. $A^{-1} I$ need not be a $*$-ideal without left distributivity even if it is nonempty.

EXAmple 4.8. Consider the $*$-continuous IL-semiring $\wp\left(T_{\Sigma}\right)$ from Example 4.2. Set

$$
I=\{\{a(b(\square), b(\square)), a(c(\square), c(\square))\}\} \downarrow \text { and } A=\{\{a(\square, \square)\}\} .
$$

Then, $I$ is a $*$-ideal and $A^{-1} I=\{\{b(\square)\},\{c(\square)\}\}$. But $A^{-1} I$ is not a $*$-ideal since it is not closed under $\cup$.

Lemma 4.9. Let $S$ be a *-continuous IL-semiring. The following holds for nonempty subsets $A, B \subseteq S$.

1. $\langle A \odot B\rangle=\langle\langle A\rangle \odot B\rangle$.

2. $\langle A \odot B\rangle=\langle A \odot\langle B\rangle\rangle$ if $S$ satisfies left distributivity.

Proof. The inclusion $\subseteq$ of 1 follows from monotonicity of $\left\langle_{-}\right\rangle$. Next, we show the reverse inclusion. Since $A \odot B \subseteq\langle A \odot B\rangle$, we have $A \subseteq\langle A \odot B\rangle B^{-1}$. By Lemma 4.7 and monotonicity of $\left\langle_{-}\right\rangle$, it holds that $\langle A\rangle \subseteq\langle A \odot B\rangle B^{-1}$. Thus, $\langle A\rangle \odot B \subseteq\langle A \odot B\rangle$ holds. Again, by monotonicity of $\left\langle_{-}\right\rangle$, the reverse inclusion holds. $B \subseteq A^{-1}\langle A \odot B\rangle$ follows from $A \odot B \subseteq\langle A \odot B\rangle$. Since $B$ is nonempty, so is $A^{-1}\langle A \odot B\rangle$. Thus, the rest of proof of 2 may be done similarly to 1 .

The equation 2 of Lemma 4.9 need not hold without left distributivity.

Example 4.10. Consider the $*$-continuous IL-semiring $\wp\left(T_{\Sigma}\right)$ from Example 4.2. Set

$$
A=\{\{a(\square, \square)\}\} \text { and } B=\{\{b(\square)\},\{c(\square)\}\} .
$$

Then, $\langle B\rangle=\{\{b(\square), c(\square)\}\} \downarrow$ and $A \odot B=\{\{a(\square, \square)\} \cdot\{b(\square)\},\{a(\square, \square\} \cdot\{c(\square)\}\}$. So, $\{a(\square, \square)\} \cdot\{b(\square), c(\square)\} \in A \odot\langle B\rangle$ though $\{a(\square, \square)\} \cdot\{b(\square), c(\square)\} \notin\langle A \odot B\rangle$.

REMARK 4.11 (Kozen (1990)). If a $*$-continuous IL-semiring $S$ satisfies left distributivity, then

$$
\langle A \odot B\rangle=\langle\langle A\rangle \odot\langle B\rangle\rangle
$$

for any nonempty subsets $A, B \subseteq S$.

We give a counter example of 2 of Lemma 4 in Furusawa and Sanda (2009), that is, for nonempty subsets $A, B$ of $*$-continuous IL-semiring $S,\langle A \odot B\rangle=\langle A \odot\langle B\rangle\rangle$ holds if $B$ is closed under + .

Example 4.12. Consider the $*$-continuous IL-semiring $\wp\left(T_{\Sigma}\right)$ from Example 4.2. Set

$$
\begin{aligned}
& A=\{\{a(\square, \square)\}\} \\
& B=\left\{L_{n} \mid n \geq 0\right\} \text { where } L_{n}=\{b(\square)\}^{(n)} \cup\{c(\square)\} .
\end{aligned}
$$

Then, $B$ is closed under $\cup$. Since $\langle B\rangle$ is closed downward under $\subseteq,\{c(\square)\} \in\langle B\rangle$ and, for all $n \geq 0,\{b(\square)\}^{(n)} \in\langle B\rangle$. Also, $\{b(\square)\}^{*} \in\langle B\rangle$ by the condition on * of $*$-ideals. Moreover, since $\langle B\rangle$ is closed under $\cup,\{b(\square)\}^{*} \cup\{c(\square)\} \in\langle B\rangle$. Therefore,

$$
\{a(\square, \square)\} \cdot\left(\{b(\square)\}^{*} \cup\{c(\square)\}\right) \in\langle A \odot\langle B\rangle\rangle .
$$


On the other hand, considering $\left\{M_{n}^{\prime} \mid n \geq 0\right\} \downarrow$ defined in Example 4.2, since

$$
\begin{aligned}
\{a(\square, \square)\} \cdot\left(\{b(\square)\}^{*} \cup\{c(\square)\}\right) & \cup(\square) \\
= & \left\{a(c(\square), c(\square)\} \cup\left\{a\left(b^{k}(\square), c(\square)\right) \mid k \geq 0\right\}\right. \\
& \cup\left\{a\left(c(\square), b^{l}(\square)\right) \mid l \geq 0\right\} \cup\left\{a\left(b^{k}(\square), b^{l}(\square)\right) \mid k, l \geq 0\right\},
\end{aligned}
$$

$\{a(\square, \square)\} \cdot\left(\{b(\square)\}^{*} \cup\{c(\square)\}\right)$ is not contained by it. Moreover, it holds that

$$
\begin{aligned}
A \odot B & =\left\{\{a(\square, \square)\} \cdot L_{n} \mid n \geq 0\right\} \\
& =\left\{M_{n} \mid n \geq 0\right\} \\
& \subseteq\left\{M_{n}^{\prime} \mid n \geq 0\right\} \downarrow
\end{aligned}
$$

Therefore, since $\left\{M_{n}^{\prime} \mid n \geq 0\right\} \downarrow$ is a $*$-ideal as we have seen in Example 4.2,

$$
\{a(\square, \square)\} \cdot\left(\{b(\square)\}^{*} \cup\{c(\square)\}\right) \notin\langle A \odot B\rangle .
$$

By the above discussion, $\langle A \odot B\rangle \neq\langle A \odot\langle B\rangle\rangle$ even though $B$ is closed under $\cup$.

\section{On Ideal Completion}

In general, the natural ordering on a $*$-continuous IL-semiring is a join semilattice but not complete as we have seen in Remark 2.5. In this section, we begin with providing a completion of $*$-continuous IL-semirings.

Let $S$ be a $*$-continuous IL-semiring and consider the poset $(\mathcal{I}(S), \subseteq)$. Then, $\langle 0\rangle$ is the least element of $\mathcal{I}(S)$ since $\langle 0\rangle=\{0\}$. For each nonempty subset $\mathcal{A} \subseteq \mathcal{I}(S)$, a $*$-ideal $\langle\bigcup \mathcal{A}\rangle$ is the least upper bound of $\mathcal{A}$. Obviously, the least upper bound of $\emptyset \subseteq \mathcal{I}(S)$ is $\langle 0\rangle$. Also, since $\langle a\rangle=\{a\} \downarrow$,

$$
a \leq b \Longleftrightarrow\langle a\rangle \subseteq\langle b\rangle
$$

holds for any $a, b \in S$. Thus, $(\mathcal{I}(S), \subseteq)$ is an ideal completion of $(S, \leq)$ with an embed$\operatorname{ding}\left\langle_{-}\right\rangle: S \rightarrow \mathcal{I}(S)$. We write $\sum \mathcal{A}$ for the least upper bound of $\mathcal{A} \subseteq \mathcal{I}(S)$. For any $I, J \in \mathcal{I}(S)$, we write $I+J$ for $\sum\{I, J\}$, and define $I \cdot J=\langle I \odot J\rangle$.

Proposition 5.1. Let $S$ be a $*$-continuous IL-semiring. For each $I \in \mathcal{I}(S)$ and $\mathcal{A} \subseteq \mathcal{I}(S)$, the following holds.

1. $\langle 0\rangle \cdot I=\langle 0\rangle$.

2. $\langle 1\rangle \cdot I=I=I \cdot\langle 1\rangle$.

3. $\left(\sum \mathcal{A}\right) \cdot I=\sum\{J \cdot I \mid J \in \mathcal{A}\}$.

Proof. 1 follows from definition of $\odot$. For 2 , first recall that $\langle 1\rangle=\{1\} \downarrow$. Then,

$$
I=\{1\} \odot I \subseteq\langle 1\rangle \odot I=(\{1\} \downarrow) \odot I \subseteq(\{1\} \odot I) \downarrow=I \downarrow=I,
$$


and so $\langle 1\rangle \odot I=I$. Similarly, $I \odot\langle 1\rangle=I$. Therefore, 2 holds. 3 holds by $\sum \mathcal{A}=\langle 0\rangle$ and 1 if $\mathcal{A}=\emptyset$. Otherwise, 3 follows from

$$
\begin{array}{rlr}
\left(\sum \mathcal{A}\right) \cdot I & =\left\langle\left(\sum \mathcal{A}\right) \odot I\right\rangle & \\
& =\langle\langle\bigcup \mathcal{A}\rangle \odot I\rangle & \\
& =\langle(\bigcup \mathcal{A}) \odot I\rangle & \\
& =\langle\bigcup\{J \odot I \mid J \in \mathcal{A}\}\rangle & \\
& =\langle\bigcup\{\langle J \odot I\rangle \mid J \in \mathcal{A}\}\rangle \quad \text { (by Lemma 4.3) } \\
& =\langle\bigcup\{J \cdot I \mid J \in \mathcal{A}\}\rangle \\
& =\sum\{J \cdot I \mid J \in \mathcal{A}\} .
\end{array}
$$

\subsection{Case with Left Distributivity}

We construct a functor from ILS $S_{+}^{*}$ to ILS ${ }_{+}^{D}$ which is a left adjoint to the inclusion functor $G_{+}: \mathrm{ILS}_{+}^{D} \rightarrow \mathrm{ILS}_{+}^{*}$.

Proposition 5.2. Let $S$ be a $*$-continuous IL-semiring satisfying left distributivity. For any $H, I, J \in \mathcal{I}(S)$ and nonempty subset $\mathcal{A} \subseteq \mathcal{I}(S)$, the following holds.

1. $(H \cdot I) \cdot J=J \cdot(I \cdot J)$.

2. $I \cdot\left(\sum \mathcal{A}\right)=\sum\{I \cdot J \mid J \in \mathcal{A}\}$.

Proof. 1 follows from

$$
\begin{aligned}
(H \cdot I) \cdot J & =\langle\langle H \odot I\rangle \odot J\rangle & \\
& =\langle(H \odot I) \odot J\rangle & \quad \text { (by } 1 \text { of Lemma 4.9) } \\
& =\langle H \odot(I \odot J)\rangle & \\
& =\langle H \odot\langle I \odot J\rangle\rangle & (\text { by } 2 \text { of Lemma 4.9) } \\
& =H \cdot(I \cdot J) . &
\end{aligned}
$$

2 is proved similarly to 3 of Proposition 5.1, using 2 of Lemma 4.9 instead of 1 of it.

Therefore, by Proposition 5.1 and 1 of Remark 2.11, $\mathcal{I}(S)$ forms a D-continuous ILsemiring satisfying left distributivity.

Let $S$ be a $*$-continuous IL-semiring satisfying left distributivity. Using $\langle x\rangle=\{x\} \downarrow$, it is verified that the mapping

$$
x \mapsto\langle x\rangle
$$

from $S$ to $\mathcal{I}(S)$ is one-to-one and preserves $+, \cdot,{ }^{*}, 0$, and 1 . Thus, this mapping is an arrow from $S$ to $\mathcal{I}(S)$ in ILS* .

Following referee's suggestion, we have omitted a transfinitely inductive construction of a *-ideal, which has been adopted by Kozen (1990), Furusawa and Sanda (2009), and in the previous version of this paper. Thus, we do not allow to prove Lemma 5.4 modifying the proof of Lemma 5 (corresponding to Lemma 5.4 in this paper) in 
Furusawa and Sanda (2009), which depends on the transfinitely inductive construction. Here, we prove the lemma according to the proof given in the referee's report.

Let $S$ and $S^{\prime}$ be *-continuous IL-semirings. Given a homomorphism $f$ from $S$ to $S^{\prime}$, we define

$$
f[A]=\{f(a) \mid a \in A\} \quad \text { and } \quad f^{-1}\left[A^{\prime}\right]=\left\{a \mid f(a) \in A^{\prime}\right\}
$$

for each $A \subseteq S$ and $A^{\prime} \subseteq S^{\prime}$, respectively. Note that $f[A] \subseteq A^{\prime}$ iff $A \subseteq f^{-1}\left[A^{\prime}\right]$. Also, note that

$$
\begin{aligned}
f[A \odot B] & =f[A] \odot f[B] \\
f[A \oplus B] & =f[A] \oplus f[B] \\
f[A \downarrow] & \subseteq f[A] \downarrow \\
f\left[A_{*}\right] & \subseteq f[A]_{*}
\end{aligned}
$$

for all $A, B \subseteq S$.

Lemma 5.3. Let $S$ and $S^{\prime}$ be *-continuous $I L$-semirings and $f: S \rightarrow S^{\prime}$ a homomorphism. Then the following holds for any $A^{\prime}, B^{\prime} \subseteq S^{\prime}$.

1. $f^{-1}\left[A^{\prime}\right] \odot f^{-1}\left[B^{\prime}\right] \subseteq f^{-1}\left[A^{\prime} \odot B^{\prime}\right]$.

2. $f^{-1}\left[A^{\prime}\right] \oplus f^{-1}\left[B^{\prime}\right] \subseteq f^{-1}\left[A^{\prime} \oplus B^{\prime}\right]$.

3. $f^{-1}\left[A^{\prime}\right] \downarrow \subseteq f^{-1}\left[A^{\prime} \downarrow\right]$.

4. $f^{-1}\left[A^{\prime}\right]_{*} \subseteq f^{-1}\left[A_{*}^{\prime}\right]$.

5. If $A^{\prime} \in \mathcal{I}\left(S^{\prime}\right)$, then $f^{-1}\left[A^{\prime}\right] \in \mathcal{I}(S)$

Proof. The first inclusion is equivalent to $f\left[f^{-1}\left[A^{\prime}\right] \odot f^{-1}\left[B^{\prime}\right]\right] \subseteq A^{\prime} \odot B^{\prime}$. Also, it holds that $f\left[f^{-1}\left[A^{\prime}\right] \odot f^{-1}\left[B^{\prime}\right]\right]=f\left[f^{-1}\left[A^{\prime}\right]\right] \odot f\left[f^{-1}\left[B^{\prime}\right]\right]$. Since $f\left[f^{-1}\left[A^{\prime}\right]\right] \subseteq A^{\prime}$ and $f\left[f^{-1}\left[B^{\prime}\right]\right] \subseteq B^{\prime}, 1$ holds. 2 is proved similarly to 1 . The third inclusion is equivalent to $f\left[f^{-1}\left[A^{\prime}\right] \downarrow\right] \subseteq A^{\prime} \downarrow$. Since $f\left[f^{-1}\left[A^{\prime}\right] \downarrow\right] \subseteq f\left[f^{-1}\left[A^{\prime}\right]\right] \downarrow$ and $f\left[f^{-1}\left[A^{\prime}\right]\right] \subseteq A^{\prime}, 3$ holds. 4 is proved similarly to 3 . It is sufficient for 5 to check nonemptiness of $f^{-1}\left[A^{\prime}\right]$ since the others are induced by 2,3 , and 4 . Suppose that $A^{\prime}$ is a $*$-ideal. Then, $0 \in A^{\prime}$. So, $f[\{0\}]=\{0\} \subseteq A^{\prime}$. Thus $\{0\} \subseteq f^{-1}\left[A^{\prime}\right]$. Therefore, $f^{-1}\left[A^{\prime}\right]$ is nonempty.

Lemma 5.4. Let $S$ and $S^{\prime}$ be *-continuous $I L$-semirings and $f: S \rightarrow S^{\prime}$ a homomorphism. For each nonempty subset $A \subseteq S$, the following holds.

$$
\begin{aligned}
f[\langle A\rangle] & \subseteq\langle f[A]\rangle \\
\langle f[\langle A\rangle]\rangle & =\langle f[A]\rangle .
\end{aligned}
$$

Proof. The first inclusion follows from

$$
\begin{aligned}
f[A] \subseteq\langle f[A]\rangle & \Longleftrightarrow A \subseteq f^{-1}[\langle f[A]\rangle] \\
& \Longleftrightarrow\langle A\rangle \subseteq f^{-1}[\langle f[A]\rangle] \quad \text { (By 5 of Lemma 5.3) } \\
& \Longleftrightarrow f[\langle A\rangle] \subseteq\langle f[A]\rangle .
\end{aligned}
$$

The inclusion $\langle f[\langle A\rangle]\rangle \subseteq\langle f[A]\rangle$ follows from the first, and the reverse inclusion follows from the monotonicity of $f$ and $\left\langle_{-}\right\rangle$. 
Let $S$ be a $*$-continuous IL-semiring, $Q$ a D-continuous IL-semiring, and $g: S \rightarrow G(Q)$ a homomorphism. By Lemma 5.4, g[I] and $g[A]$ generate the same $*$-ideal if $I \in \mathcal{I}(S)$ is generated by $A \subseteq S$. For $I \in \mathcal{I}(S)$, the least upper bound of $g[I]$ exists in $Q$, which is denoted by $\sum g[I]$, since the least upper bound of any subset of $Q$ exists in $Q$.

Lemma 5.5. Let $S$ be a $*$-continuous $I L$-semiring, $Q$ a D-continuous IL-semiring, and $g: S \rightarrow G(Q)$ a homomorphism. If $I \in \mathcal{I}(S), \sum g[I]=\sum g[A]$ for any generating set $A$ of $I$.

Proof. By Lemma 5.4,

$$
\langle g[I]\rangle=\langle g[\langle A\rangle]\rangle=\langle g[A]\rangle
$$

for any generating set $A$ of $I$. Since

$$
\langle g[I]\rangle=\langle g[A]\rangle \subseteq\left\langle\sum g[A]\right\rangle=\left(\sum g[A]\right) \downarrow
$$

$\sum g[A]$ is an upper bound of $g[I]$. By $g[A] \subseteq g[I], \sum g[A] \leq \sum g[I]$. Thus, since $\sum g[I]$ is the least upper bound of $g[I], \sum g[A]=\sum g[I]$.

Let $S$ be a $*$-continuous IL-semiring satisfying left distributivity and $Q$ a Dcontinuous IL-semiring satisfying left distributivity. Define the map $\widehat{g}: \mathcal{I}(S) \rightarrow Q$ by

$$
\widehat{g}(I)=\sum g[I]
$$

for a homomorphism $g: S \rightarrow G_{+}(Q)$.

Proposition 5.6. The map $\widehat{g}$ preserves $\sum, \cdot, 0$ and 1 .

Proof. For $I, J \in \mathcal{I}(S)$,

$$
\begin{array}{rlrl}
\widehat{g}(I \cdot J) & =\sum g[I \cdot J] & \\
& =\sum g[\langle I \odot J\rangle] & \\
& =\sum g[I \odot J] & & \text { (by Lemma 5.5) } \\
& =\sum g[I] \odot g[J] & \\
& =\left(\sum g[I]\right) \cdot\left(\sum g[J]\right) & \text { (by left distributivity) } \\
& =\widehat{g}(I) \cdot \widehat{g}(J) . &
\end{array}
$$

Also, we have

$$
\widehat{g}(\langle 1\rangle)=\sum g[\langle 1\rangle]=\sum g[\{1\}]=1
$$

and

$$
\widehat{g}(\langle 0\rangle)=\sum g[\langle 0\rangle]=\sum g[\{0\}]=0 .
$$


Note that $\sum(\bigcup \mathcal{B})=\sum\left\{\sum_{A} B \mid B \in \mathcal{B}\right\}$ for a subset $\mathcal{B}$ of the powerset $\wp(Q)$. Then, for nonempty subset $\mathcal{A} \subseteq \mathcal{I}(S)$,

$$
\begin{aligned}
\widehat{g}\left(\sum \mathcal{A}\right) & =\sum g\left[\sum \mathcal{A}\right] \\
& =\sum g[\langle\bigcup \mathcal{A}\rangle] \\
& =\sum g[\bigcup \mathcal{A}] \\
& =\sum(\bigcup\{g[I] \mid I \in \mathcal{A}\}) \\
& =\sum\left\{\sum g[I] \mid I \in \mathcal{A}\right\} \\
& =\sum\{\widehat{g}[I] \mid I \in \mathcal{A}\} .
\end{aligned}
$$

The equation holds even in the case of $\mathcal{A}=\emptyset$ since $\sum \mathcal{A}=\langle 0\rangle$ and it has been shown that $\widehat{g}$ preserves 0

THEOREM 5.7. Let $S$ be a *-continuous IL-semiring satisfying left distributivity and $Q$ a D-continuous IL-semiring satisfying left distributivity. For a homomorphism $g: S \rightarrow G_{+}(Q), \widehat{g}$ is a unique completely join-preserving homomorphism from $\mathcal{I}(S)$ to $Q$ such that $g=\widehat{g} \circ\langle-\rangle$.

Proof. For each $a \in S$, we have

$$
\widehat{g}(\langle a\rangle)=\sum g[\langle a\rangle]=\sum g[\{a\}]=g(a)
$$

by Lemma 5.5. Assume that a completely join-preserving homomorphism $f$ from $\mathcal{I}(S)$ to $Q$ satisfies $g=f \circ\langle-\rangle$. Then, it holds that

$$
\begin{aligned}
\widehat{g}(I) & =\sum g[I] \\
& =\sum\{g(a) \mid a \in I\} \\
& =\sum\{f(\langle a\rangle) \mid a \in I\} \quad \text { (by assumption) } \\
& =f\left(\sum\{\langle a\rangle \mid a \in I\}\right) \\
& =f(I)
\end{aligned}
$$

for each $I \in \mathcal{I}(S)$. Thus, $\widehat{g}=f$.

For a $*$-continuous IL-semiring $S$ satisfying left distributivity and a homomorphism $h: S \rightarrow S^{\prime}$, we define

$$
F_{+}(S)=\mathcal{I}(S) \quad \text { and } \quad F_{+}(h)=\widehat{\langle-\rangle \circ h},
$$

respectively. Then, $F_{+}$is a functor from ILS* to ILS $S_{+}^{D}$. It is immediate from Theorem 5.7 that the following holds.

CoROLLARY 5.8. The functor $F_{+}: \mathrm{ILS}_{+}^{*} \rightarrow \mathrm{ILS}_{+}^{D}$ is a left adjoint to the inclusion functor $G_{+}: \mathrm{ILS}_{+}^{D} \rightarrow \mathrm{ILS}_{+}^{*}$. 
$\mathrm{ILS}_{0,+}^{*}$ is a full subcategory of ILS $S_{+}^{*}$. The inclusion functor from ILS $0_{0,+}^{*}$ to $\mathrm{ILS}_{+}^{*}$ is denoted by $E^{*}$. Also, $\operatorname{ILS}_{0,+}^{D}$ is a full subcategory of $\operatorname{ILS}_{+}^{D}$. The inclusion functor from $\mathrm{ILS}_{0,+}^{D}$ to $\mathrm{ILS}_{+}^{D}$ is denoted by $E^{D}$. Note that

$$
\begin{aligned}
E^{*}(S) & =S \\
E^{D}(Q) & =Q \\
\operatorname{ILS}_{0,+}^{*}\left(S, S^{\prime}\right) & =\operatorname{ILS}_{+}^{*}\left(E^{*}(S), E^{*}\left(S^{\prime}\right)\right) \\
\operatorname{ILS}_{0,+}^{D}\left(Q, Q^{\prime}\right) & =\operatorname{ILS}_{+}^{D}\left(E^{D}(Q), E^{D}\left(Q^{\prime}\right)\right)
\end{aligned}
$$

for any objects $S, S^{\prime}$ of $\mathrm{ILS}_{0,+}^{*}$ and $Q, Q^{\prime}$ of $\mathrm{ILS}_{0,+}^{D}$. Also, note that

$$
G_{0,+}=G_{+} \circ E^{D} \text {. }
$$

Proposition 5.9. Let $S$ be a $*$-continuous IL-semiring. If $S$ satisfies the right zero law, $I \cdot\langle 0\rangle=\langle 0\rangle$ for each $I \in \mathcal{I}(S)$.

Proof. Since $\langle 0\rangle=\{0\}$ and $S$ satisfies the right zero law, we have

$$
I \cdot\langle 0\rangle=\langle I \odot\{0\}\rangle=\langle 0\rangle .
$$

Define $F_{0,+}=F_{+} \circ E^{*}$. Then, by Proposition $5.9, F_{0,+}$ is a functor from $\mathrm{ILS}_{0,+}^{*}$ to $\mathrm{ILS}_{0,+}^{D} \cdot$

THeOREM 5.10. The functor $F_{0,+}: \mathrm{ILS}_{0,+}^{*} \rightarrow \mathrm{ILS}_{0,+}^{D}$ is a left adjoint to the inclusion functor $G_{0,+}$ : ILS $\mathrm{IL}_{0,+}^{D} \rightarrow \mathrm{ILS}_{0,+}^{*}$. from

Proof. Let $S$ be an object of $\mathrm{ILS}_{0,+}^{*}$ and $Q$ an object of ILS ${ }_{0,+}^{D}$. Then, it follows

$$
\begin{aligned}
\operatorname{ILS}_{0,+}^{*}\left(S, G_{0,+}(Q)\right) & =\operatorname{ILS}_{+}^{*}\left(E^{*}(S), G_{+}\left(E^{D}(Q)\right)\right) \\
& \cong \operatorname{ILS}_{+}^{D}\left(F_{+}\left(E^{*}(S)\right), E^{D}(Q)\right) \quad \text { (by Corollary 5.8) } \\
& =\operatorname{ILS}_{0,+}^{D}\left(F_{0,+}(S), Q\right) .
\end{aligned}
$$

\subsection{Case without Left Distributivity}

We give a counter example of 4 of Proposition 2 in Furusawa and Sanda (2009), that is, for each $*$-continuous IL-semiring $S, I \in \mathcal{I}(S)$, and $\mathcal{A} \subseteq \mathcal{I}(S)$,

$$
I \cdot\left(\sum \mathcal{A}\right)=\sum\{I \cdot J \mid J \in \mathcal{A}\}
$$

holds if $\mathcal{A}$ is directed with respect to $\subseteq$.

EXAmPle 5.11. Consider the $*$-continuous IL-semiring $\wp\left(T_{\Sigma}\right)$ from Example 4.2. Set

$$
\begin{aligned}
& I=\langle\{a(\square, \square)\}\rangle \\
& \mathcal{A}=\left\{J_{n} \mid n \geq 0\right\} \text { where } J_{n}=\left\langle\{b(\square)\}^{(n)} \cup\{c(\square)\}\right\rangle .
\end{aligned}
$$


Note that $I=\{\emptyset,\{a(\square, \square)\}\}$ and $J_{n}=\left\langle L_{n}\right\rangle$, where $L_{n}$ is from Example 4.12. Then, $\mathcal{A}$ is directed with respect to $\subseteq$. Since $\sum \mathcal{A}$ is closed downward under $\subseteq,\{c(\square)\} \in \sum \mathcal{A}$ and, for all $n \geq 0,\{b(\square)\}^{(n)} \in \sum \mathcal{A}$. Also, $\{b(\square)\}^{*} \in \sum \mathcal{A}$ by the condition on * of $*$-ideals. Moreover, since $\sum \mathcal{A}$ is closed under $\cup,\{b(\square)\}^{*} \cup\{c(\square)\} \in \sum \mathcal{A}$. Thus, $\{a(\square, \square)\} \cdot\left(\{b(\square)\}^{*} \cup\{c(\square)\}\right) \in I \odot \sum \mathcal{A}$. Therefore, since $I \odot\left(\sum \mathcal{A}\right) \subseteq I \cdot\left(\sum \mathcal{A}\right)$, it holds that

$$
\{a(\square, \square)\} \cdot\left(\{b(\square)\}^{*} \cup\{c(\square)\}\right) \in I \cdot\left(\sum \mathcal{A}\right) .
$$

On the other hand, $\{a(\square, \square)\} \cdot\left(\{b(\square)\}^{*} \cup\{c(\square)\}\right) \notin\left\{M_{n}^{\prime} \mid n \geq 0\right\} \downarrow$ as we have seen in Example 4.12. Since $\{b(\square)\}^{(n)} \cup\{c(\square)\}$ is the greatest language in $J_{n}$ and

$$
\begin{aligned}
I \odot J_{n} & =\{\emptyset,\{a(\square, \square)\}\} \odot J_{n} \\
& =\{\emptyset\} \cup\left\{\{a(\square, \square)\} \cdot L \mid L \in J_{n}\right\} \quad \\
& =\left\{\{a(\square, \square)\} \cdot L \mid L \in J_{n}\right\} \quad\left(\text { by } \emptyset \in J_{n}\right),
\end{aligned}
$$

$L \in I \odot J_{n}$ implies $L \subseteq M_{n}$, where $M_{n}$ is defined in Example 4.2. So, for all $n \geq 0$, $I \odot J_{n} \subseteq\left\{M_{n}^{\prime} \mid n \geq 0\right\} \downarrow$. Thus, $\sum\{I \cdot J \mid J \in \mathcal{A}\} \subseteq\left\{M_{n}^{\prime} \mid n \geq 0\right\} \downarrow$. Therefore,

$$
\{a(\square, \square)\} \cdot\left(\{b(\square)\}^{*} \cup\{c(\square)\}\right) \notin \sum\{I \cdot J \mid J \in \mathcal{A}\} .
$$

This example shows that the set of $*$-ideals of a $*$-continuous IL-semiring (satisfying the right zero law) need not be a D-continuous IL-semirings (satisfying the right zero law). So, this construction provides neither a functor from ILS* to ILS ${ }^{D}$ nor one from ILS* to $\mathrm{ILS}_{0}^{D}$.

\section{Conclusion}

Furusawa and Sanda (2009) reported that left adjoints to the following inclusion functors

$$
\begin{aligned}
& \mathrm{ILS}^{*} \longleftarrow \mathrm{ILS}^{D}
\end{aligned}
$$

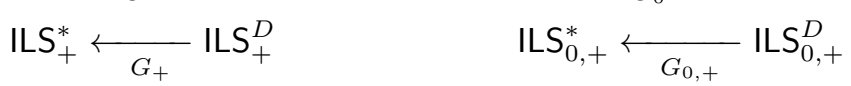

are constructed via an ideal completion. However, two of these are wrong. In this paper we have shown that

1. left adjoints to the inclusion functors are constructed via the ideal completion in the case of satisfying left distributivity,

2. otherwise, even functors in the opposite direction to the inclusion functors cannot be constructed via the ideal completion.

For the second, we have given an example using a $*$-continuous IL-semiring over a monodic tree languages.

In Proposition 5.2 it has been shown that the binary operation - on $\mathcal{I}(S)$ of a *continuous IL-semiring $S$ satisfying left distributivity is associative. This fact arises the obvious question whether a $*$-continuous IL-semiring $S$ such that the binary operation . 
on $\mathcal{I}(S)$ is not associative exists. If such a $*$-continuous IL-semiring exists, it is another (yet more essential than Example 5.11) evidence to show that we can not obtain a functor from ILS* to ILS ${ }^{D}$ via the ideal completion. However, this question is still open.

\section{Acknowledgement}

The authors wish to thank Wolfram Kahl for his careful reading of examples. The authors also have benefited from anonymous referee's constructive suggestions to the previous version of this paper. This work was supported in part by Grants-in-Aid for Scientific Research (C) 22500016 and Grant-in-Aid for JSPS Fellows 22-7560 from Japan Society for the Promotion of Science (JSPS).

\section{References}

Barr, M., Wells, C. (1999). Category Theory for Computing Science, Third Edition. Les Publications.

Conway, J.H. (1971). Regular Algebra and Finite Machines. Chapman and Hall.

Furusawa, H., Nishizawa, K., Tsumagari, N. (2009). Multirelational Models of Lazy, Monodic Tree, and Probabilistic Kleene Algebras. Bulletin of Informatics and $\mathrm{Cy}$ bernetics, 41: 11-24.

Furusawa, H. and Sanda, F. (2009). *-Continuous Idempotent Left Semirings and Their Ideal Completion. In: Berghammer, R., Jaoua, A., Möller, B. (eds.) RelMiCS/AKA 2009. LNCS, vol. 5827, pp. 119-133. Springer.

Kozen, D. (1990). On Kleene Algebras and Closed Semirings. In: Rovan, B. (ed.) MFCS 1990. LNCS, vol. 452, pp. 26-47. Springer.

Kozen, D. (1994). A Completeness Theorem for Kleene Algebras and the Algebra of Regular Events. Information and Computation, 110: 366-390.

Mac Lane, S. (1998). Categories for the Working Mathematician, Second Edition. Springer.

McIver, A., Weber, T. (2005). Towards Automated Proof Support for Probabilistic Distributed Systems. In: Sutcliffe, G., Voronkov, A. (eds.) LPAR 2005. LNCS(LNAI), vol. 3835, pp. 534-548. Springer.

Möller, B. (2004). Lazy Kleene Algebra. In: Kozen, D. (ed.) MPC 2004. LNCS, vol. 3125, pp. 252-273. Springer.

Nishizawa, K., Tsumagari, N., Furusawa, H. (2009). The cube of Kleene algebras and the triangular prism of multirelations. In: Berghammer, R., Jaoua, A., Möller, B. (eds.) RelMiCS/AKA 2009. LNCS, vol. 5827, pp.276-290. Springer.

Takai,T., Furusawa, H. (2006). Monodic Tree Kleene Algebra. In: Schmidt, R.A. (ed.) RelMiCS/AKA 2006. LNCS, vol. 4136, pp. 402-416. Springer. 